\title{
Mechanisms of austenite-martensite transition
}

\author{
V. Paidar ${ }^{\mathrm{a}}$ \\ Institute of Physics AS CR, 18221 Praha 8, Czech Republic
}

\begin{abstract}
Martensitic transformations originate from the interaction of the short-wave (atomic plane shuffling) and long-wave (homogeneous deformation) processes. The mutual role of these movements is examined with the aim to elucidate formation mechanisms of the structures with lower symmetry at the atomic level. Generic many-body potentials differing in the stability of basic simple structures is employed in the calculations of the system energies in the course of phase transition.
\end{abstract}

\section{Introduction}

An important class of so-called smart materials embraces also the materials that possess the property of shape memory. This behaviour is directly associated with displacive or diffusionless phase transformations. Two processes at the atomic level play a fundamental role in shape memory materials, namely shuffling of atomic planes and homogeneous deformation of shear type (see also [1]). These intrinsic parts of phase transformation mechanisms from a bcc-type austenite to a close-packed martensite are manifested in phonon anomalies and in softening of $\mathrm{c}^{\prime}$ elastic constant describing the shear deformation on the (101) plane in the [ 101 ]direction [2]. Repeated shuffling on every other parallel atomic plane generates close-packed topology but the bcc atomic triangles remain isosceles and are not equilateral as in the hcp or fcc structures. A contraction of about $11 \%$ in the [010] cubic direction together with the extension of $9 \%$ in the [ $\overline{101}$ ] perpendicular direction ensures the correct dimensions of the hcp basal plane. Moreover, the interplanar separation of the basal planes is slightly larger (about 3\%) in hcp or fcc than that of the (101) bcc planes. The repeated shuffling matches the frozen phonon mode with the wave vector at the Brillouin zone boundary.

Several transformation paths to the close-packed structures are known. Those are uniaxial deformations preserving the atomic volume, i.e. the uniaxial contraction supplemented by the biaxial planar extension. In the case of four-fold [010] cubic axis, the tetragonal Bain path leads from the bcc to fcc structures [3]. For the [111] cubic axis a trigonal path arises connecting the bcc, simple cubic and fcc structures. The fcc structure can be also created by the shear on the (101) bcc plane, so called Zener path [4]. The hcp structure is formed by the alternate shuffling of the (101) bcc planes that is accompanied by the additional homogeneous deformation needed also for the Zener path.

The shape memory materials are often multi-component ordered alloys. However, this type of complexity is disregarded in our simplified generic approach. We consider the systems described by the manybody potentials differing only in one parameter representing the materials with different degree of stability of the initial (austenitic) structure.

The change of the bcc structure by pure displacements into close-packed stacking when the atoms from the upper plane are situated at the centres of the atomic triangles of the underlying plane is not sufficient for the formation of a metastable martensitic structure [5]. Additional deformation is required to lower the energy of the initial bcc-type phase. In the present paper we will first examine transition paths between austenite and martensite and then calculate the system energies along the transformation trajectory in a simple model where the interatomic forces are characterized by a many-body potential.

\footnotetext{
${ }^{a}$ e-mail: paidarefzu.cz
}

This is an Open Access article distributed under the terms of the Creative Commons Attribution-Noncommercial License (http://creativecommons.org/licenses/by-nc/3.0/), which permits unrestricted use, distribution, and reproduction in any noncommercial medium, provided the original work is properly cited. 


\section{Description of interatomic forces}

The exponential form of many-body potentials [6] with only five parameters was chosen for our model of interatomic forces. The total cohesive energy is expressed as a sum over all atoms in the system

$$
E=-\sum_{i}\left(\begin{array}{c}
{ }^{i} \\
b
\end{array}+\Xi_{r}^{i}\right)
$$

It is composed of the bonding contributions based on the density of electron states with the embedding function of Finnis-Sinclair type [7]

$$
E_{b}^{i}=-\left\{\sum_{i} \xi^{2} \exp [-2 q(\underbrace{i j}_{0}-1)]\right\}^{1 / 2}
$$

and contributions of the repulsive forces due to atom cores represented by the pairwise potential of Born-Mayer type

$$
E_{r}^{i}=\sum_{j} \exp \left[-\left(\frac{\dot{i j}}{\dot{j}_{0}}-1\right)\right] .
$$

The energy is thus given by four adjustable parameters $\xi, A, p$ and $q$. The interatomic distance between the atoms $\mathrm{i}$ and $\mathrm{j}$ is $r_{\mathrm{ij}}$.

The potential parameters are fitted to the cohesive energy and lattice parameter to obtain reasonable energy and length scaling. The other two parameters $p$ and $q$ in exponents are altered to modify the potential properties. Fitting was performed to the cohesive energy of $2.95 \mathrm{eV}$ and the lattice parameter of $0.409 \mathrm{~nm}$ (corresponding to silver) for all chosen values of $q . p$ was kept constant equal to 9 and $q$ varies between 1 and 3 . The fifth parameter $r_{0}$ is equal to the first neighbour separation in the fcc lattice.

Both the bonding, $E_{b}{ }^{i}$, and repulsive, $E_{r}^{i}$, energy contributions for an $i$-atom are cut off at the distance corresponding to the $4^{\text {th }}$ neighbours of the fcc lattice. Between the $3^{\text {rd }}$ and $4^{\text {th }}$ neighbours, the exponential expressions $E_{b}{ }^{i}$ and $E_{r}{ }^{i}$ (eqs. (2) and (3)) are replaced by the fifth order polynomials. The polynomial coefficients are fitted to obtain continuity of the values and first and second derivatives at the point of line-up (the $3^{\text {rd }}$ neighbours) and truncation to zero at the cut-off distance (the $4^{\text {th }}$ neighbours)

The energies are calculated per one atom and are expressed in $\mathrm{eV}$. The displacive structures with varying displacements of atomic planes are commonly characterized by the surface energies (the energies per unit plane area) but since only three-dimensional homogeneous structures will be considered in this paper, all the structures can be characterized by the energies per one atom.

\section{Transformation paths}

Two types of planar defects can be distinguished: stacking faults when one half of the crystal is displaced with respect to the other half and atomic plane shuffling when only one atomic plane is displaced while all the other planes remain in their initial positions. No local minima corresponding to metastable stacking faults on the $\{101\}$ planes in bcc crystals were reported even for different interatomic potentials [8]. Similarly, we have not found any local minima of possible metastable structures for the shuffling of a single atomic plane [5]. However, local minima of new stable structures can appear in three dimensional structures when the pure displacive processes are combined with additional deformation.

As already mentioned above, the additional deformation preserving atomic volume that generate closepacked structures is composed of the contraction of the pairs of the second bcc neighbours in the [010] direction plus the extension in the [ $\overline{101}]$ direction both in the (101) plane of displacements completed by the extension in the direction perpendicular to the (101) planes. Nevertheless, the last deformation is smaller than the former two component deformations, and this is why we will consider two following cases: full three-dimensional deformation and only biaxial planar deformation combining the contraction with the extension. The latter 
deformations can be described as simple shear on the plane perpendicular to (101). In such a way we arrive to the transformation path proposed by Burgers [9].

\subsection{Burgers path}

Contrary to the Bain path that consists only of homogeneous tetragonal deformation, the Burgers path is composed of two processes: atomic plane shuffling plus shear deformation. The shuffling takes place on the most densely packed (101) bcc atomic plane and the shear is deduced from the bcc twinning in the $<111>$ direction on the $\{121\}$ plane perpendicular to the (101) shuffling plane (see Fig. 1).

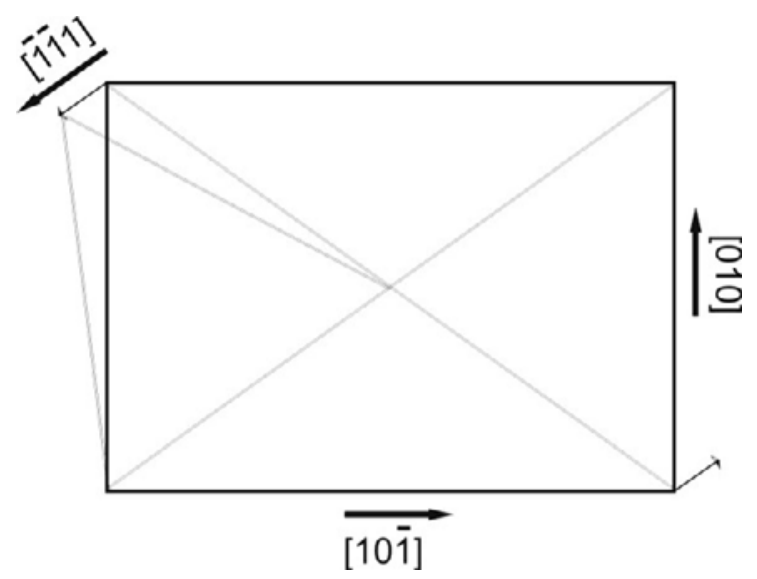

Fig. 1. Shear deformation transforming the bcc isosceles triangle formed by two bonds between the first neighbours in the $<111>$ directions and one bond between the second neighbours in the [010] direction into another isosceles triangle formed by one bond between the first neighbours and two new bonds shorter than the second neighbour bond.

Let us compare in Table 1 the dimensions of the orthorhombic lattice cells derived from the bcc austenite, i.e. bcc itself, hcp and shear cell created by the shear along the bcc close-packed [ $\overline{1} 11$ ] direction in the intersection of the (101) shuffling and ( $\overline{1} 21)$ shearing planes.

Table 1. Comparison of $2 \mathrm{H}$ martensite with the structures derived from the bcc austenite.

\begin{tabular}{|c|c|c|c|c|}
\hline & $\mathrm{a}$ & $\mathrm{b}$ & $\mathrm{c}$ & $\Delta \%$ \\
\hline $\mathrm{Cu}-27,14 \mathrm{Al}-3,64 \mathrm{Ni}$ & 0.43897 & 0.53425 & 0.42245 & \\
\hline $\mathrm{bcc}$ & 0.412237 & 0.582991 & 0.412237 & 6.49 \\
\hline hcp & 0.449801 & 0.519386 & 0.424077 & 2.16 \\
\hline shear & 0.446041 & 0.538807 & 0.412237 & 1.75 \\
\hline
\end{tabular}

The experimental values of the lattice parameters for $2 \mathrm{H}$ martensite of $\mathrm{Cu}-27,14 \mathrm{Al}-3,64 \mathrm{Ni}$ alloy are taken from [10]. They are compared with the bcc austenite with the orthorhombic dimensions $a_{b} \sqrt{2}, 2 a_{b}, a_{b} \sqrt{ } 2$, the hcp cell $a_{h} \sqrt{ } 3,2 a_{h}, 2 a_{h} \sqrt{ } 2 / \sqrt{ } 3$, where $a_{h}=a_{b} 2^{-1 / 6}$ and finally, with the shear cell $a_{b} \sqrt{ } 96 / \sqrt{ } 41, a_{b} \sqrt{ } 41 / \sqrt{ } 12, a_{b} \sqrt{ } 2$. Medium deviations of the considered structures from the observed $2 \mathrm{H}$ martensite, $\Delta=\sqrt{ }\left(\left(\Delta_{\mathrm{a}}^{2}+\Delta_{\mathrm{b}}{ }^{2}+\Delta_{\mathrm{c}}{ }^{2}\right) / 3\right)$, are compared in the last column of Table 1. All the considered structures have the same atomic volume.

\section{Energies of transformations}

It is shown in Fig. 2a that the bcc structure is more stable for small $q$ while the hcp is more stable for $q$ larger than 2 (see also [11]). The energies for three transformation mechanisms are compared in Fig. 2b-d for three different potentials with $q=1,2$ and 3 .

There are no additional minima for alternate shuffling without additional deformation for all three potentials (dashed lines in Fig. 2b-d). The energy grows from the zero value of the initial bcc structure up to the 
maximum corresponding to a saddle point on two dimensional plot for a complete $\gamma$-surface of shuffling with arbitrary displacement vector on the (101) plane. The additional deformation reaches the close-packed structure at $\mathbf{b} / 6$ and then continues to grow proportionally to the shuffling displacement in the [ $\overline{101}$ ] direction. Notice that the energy curves of shear and full deformations (dot-dashed and full lines in Fig. 2b-d) start to deviate not until larger displacements.

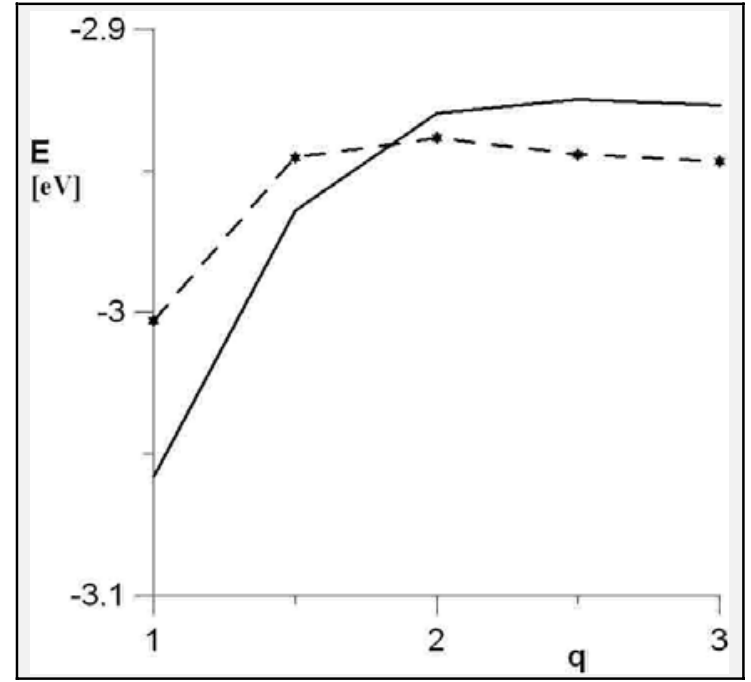

a)

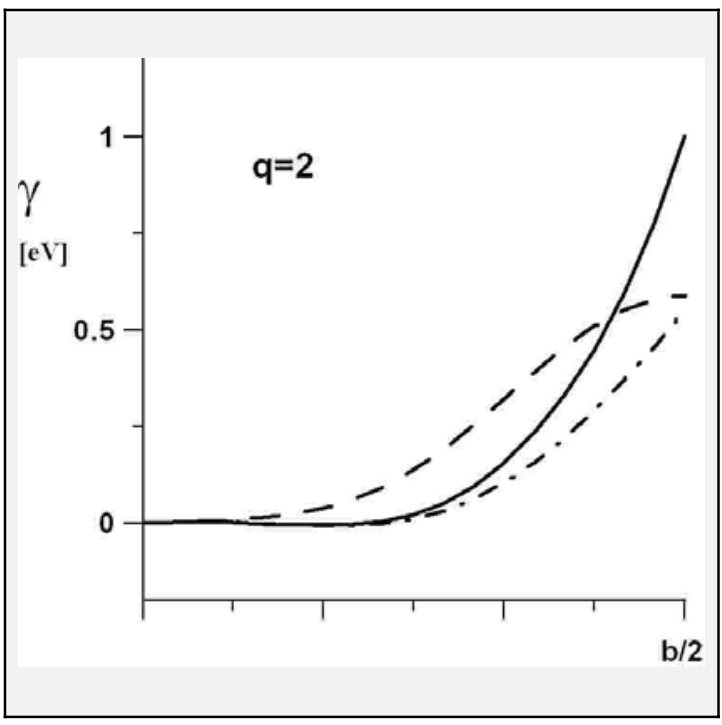

c)

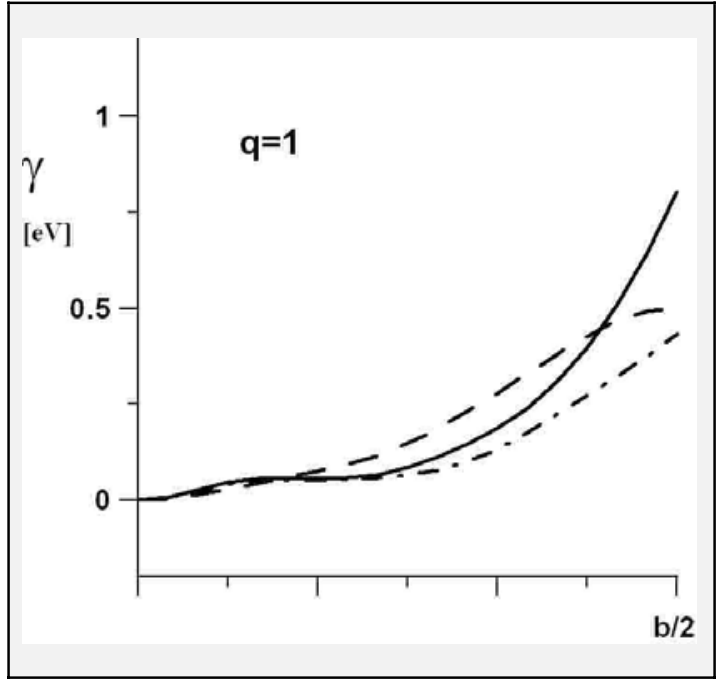

b)

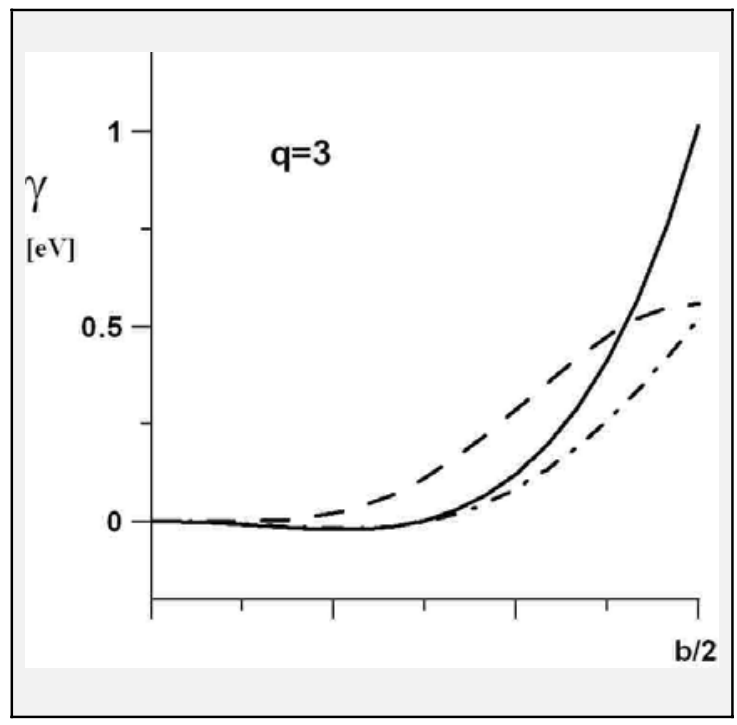

d)

Fig. 2. Calculated energies a) of the bcc austenite (full line) and hcp martensite (dashed line), b-d) along the transformation paths for only shuffling of atomic planes (dashed lines), shuffling together with shear deformation (dot-dashed lines), together with three-dimensional deformation (full lines) for three many-body potentials $q=1-3$. Vector $\mathbf{b}$ is the lattice period vector in the [ $\overline{1} 01]$ direction.

\section{Discussion}

It has been demonstrated in the previous section that the additional deformation plays an important role in the phase transitions. The displacive process associated with the short-range phonons is not sufficient for the 
formation of close-packed structure from the bcc-austenitic phase at least for the selected type of interatomic forces. On the other hand, the shear deformation creates the structures with lower energies and, moreover, the deviation form the experimentally observed structure of $2 \mathrm{H}$ martensite is closer to this sheared structure than to the ideal hcp structure (see Table 1). The shear process in the $<111>$ direction on $\{121\}$ is certainly less demanding on atomic rearrangements than the full deformation in three different directions. The magnitude of biaxial deformation required by the hcp structure is larger than observed experimentally. Nevertheless, the observed interplanar atomic spacing is larger indicating that a supplementary relaxation of (101) bcc planes occurs.

It should be noted that the transformation path considered here is slightly different from that proposed by Burgers [9]. The shear of about 0.221 leads to the angle of $60^{\circ}$ while in the isosceles triangle we have $62^{\circ}$. However, when the angle between the first bcc neighbours of $70^{\circ}$ is decreased to $60^{\circ}$, the other angle grows from $55^{\circ}$ to $64^{\circ}$. Simultaneously, decreasing the length of the second neighbour bond by the [ $\left.\overline{1} 11\right](\overline{1} 21)$ shear deformation beyond the shear of 0.177 increases unduly the length of the initially first neighbour bond. This is why we took the isosceles triangle as a better choice.

\section{Conclusions}

It has been shown that the martensitic structures can be formed on the atomic level from the bcc-type austenite by the combination of atomic plane alternate shuffling and shear deformation of the type proposed originally by Burgers.

The author is grateful to the Grant Agency of the Academy of Sciences of the Czech Republic, contract No. IAA100100920, and to the COST program P19 OC09014 for financial support.

\section{References}

[1] V. Paidar, Mater. Sci. Eng. A 481-482, 243 (2008).

[2] A. Planes and L. Manosa, in Solid State Physics, edited by H. Ehrenreich and F. Spaepen (Academic Press, 2001), Vol. 55, p. 159.

[3] E.C. Bain, Trans. AIME 70, 25 (1924).

[4] C. Zener, Phys. Rev. 71, 846 (1947).

[5] V. Paidar and A. Ostapovets, Solid State Phenomena 150, 159 (2009).

[6] V. Rosato, M. Guillopé, and B. Legrand, Phil. Mag. A 59 (2), 321 (1989).

[7] M. W. Finnis and J. E. Sinclair, Phil. Mag. A 50 (1), 45 (1984).

[8] V. Vitek, Phil. Mag. A 18, 773 (1968); V. Paidar and V. Vitek, in Intermetallic Compounds, Progress, edited by J.H. Westbrook and R.L. Fleischer (John Wiley, Chichester, 2002), Vol. 3, p. 437.

[9] W.G. Burgers, Physica 1, 561 (1934).

[10] J. Ye, M. Tokonami, and K. Otsuka, Metall. Trans. 21A, 2669 (1990).

[11] A. Ostapovets and V. Paidar, Mater. Sci. Forum 567-8, 69 (2007). 